\title{
Deposition of sediment from suspension in emergent vegetation
}

\author{
RG Sharpe and CS James* \\ Centre for Water in the Environment, School of Civil \& Environmental Engineering, University of the Witwatersrand, \\ Private Bag 3, Wits 2050, South Africa
}

\begin{abstract}
Emergent instream vegetation influences the transport and deposition of suspended sediment in rivers, and hence their morphology and nutrient dynamics. An experimental laboratory study has shown how emergent vegetation stems promote sediment deposition. The suspended transport and the extent of longitudinal deposits from suspension within emergent stems is enhanced by increased flow depth and reduced by increased sediment grain size and stem density. The shear zone between longitudinal vegetation strips and adjacent unvegetated channel flow induces diffusion of sediment into the vegetated zone. The transverse extent of the resulting deposit is enhanced by increased flow depth and stem density, and reduced by increased sediment grain size. Values of sediment diffusivity for the two experimental situations were inferred by application of two-dimensional formulations of the diffusion-convection equation. These applications indicate that vertical diffusivity is considerably reduced and transverse diffusivity in the shear zone considerably increased by the stems.
\end{abstract}

Keywords: sediment deposition; suspended sediment; emergent vegetation; sediment diffusivity

\section{Notation}

$a$ projected plant area per unit volume

$a$ height above bed for reference concentration

$C$ suspended sediment concentration

$C_{a}$ reference level suspended sediment concentration

$C_{d}^{a}$ drag coefficient of stems

$d$ stem diameter

$D$ flow depth

$p$ probability of deposition

$s$ stem spacing

$u$ velocity in subscripted direction

$U$ mean flow velocity

$u_{*}$ shear velocity

$w$ settling velocity

longitudinal direction

vertical direction

transverse direction

$\alpha$ scale factor in transverse diffusivity relationship

$\beta$ scale factor in transverse diffusivity relationship

$\beta$ proportionality between sediment diffusivity and eddy viscosity

$\varepsilon$ diffusivity in subscripted direction

$\kappa$ Von Karman constant

$v_{t}$ eddy viscosity

\section{Introduction}

Vegetation is an integral feature of many rivers and has a strong influence on their physical and ecological functioning through its interaction with local hydraulics and sediment dynamics (James et al., 2001). The occurrence of instream vegetation is associated with the distribution of deposited sediment, which

* To whom all correspondence should be addressed.

푱 +2711 717-7115; fax: +2711 339-1762; e-mail: CSJ@civil.wits.ac.za Received 6 June 2005; accepted in revised form 17 January 2006. is itself influenced by the vegetation through its effect on the local hydraulics and hence the movement, storage and stabilisation of sediment. The growth of vegetation is also determined by nutrient supply, which is associated at least in part with fine sediment. Effective environmental management of rivers therefore requires understanding of, and the ability to predict, the influence of vegetation on local hydraulics and sediment behaviour. In particular, quantitative descriptions are required of the ingress, movement and deposition of suspended sediment in vegetation stands.

The distribution of sediment concentration $(C)$ in suspended transport may be described by the diffusion-convection equation (e.g. Graf, 1971):

$$
\frac{\partial C}{\partial t}=-u_{i} \frac{\partial C}{\partial x_{i}}+\frac{\partial}{\partial x_{i}}\left(\varepsilon_{i} \frac{\partial C}{\partial x_{i}}\right)
$$

where:

$t$ is time

$u_{i}$ are velocity components in directions $x_{i}$

$\varepsilon_{i}$ are the sediment diffusivities in the corresponding

directions.

The rate of deposition at any location on the bed can be described by imposing the boundary condition (James, 1985):

$$
\varepsilon_{y} \frac{\partial C}{\partial y}+(1-p) w C=0
$$

where:

$y$ is the vertical direction

$w$ is the particle settling velocity (assumed to represent $-u_{v}$ ) $p$ is the probability that a particle reaching the bed will be permanently deposited.

Solution of Eqs. (1) and (2) to predict sediment concentration and deposition distribution patterns requires knowledge of $u_{i}$ and $\varepsilon_{i}$ values. The most common application is for determination of longitudinal suspended sediment transport rates in unvegetated 
channels under equilibrium conditions. In such cases the vertical velocity is represented by the particle settling velocity (as in Eq. (2)) and the vertical variation of longitudinal velocity by a logarithmic distribution. The sediment diffusivity can be related to the kinematic eddy viscosity of the flow, such as through Eqs. (9) and (10). For equilibrium conditions in unobstructed flow the eddy viscosity may be assumed to vary with distance from the bed in a regular way, such as with constant, parabolic or a combination of constant and parabolic distributions (e.g. Van Rijn, 1982). For longitudinal flow through emergent vegetation stems the velocity is almost constant vertically, and can be estimated by appropriate vegetative resistance equations (e.g. James et al., 2004), but little is known of the vertical eddy viscosity and its distribution. Lopez and Garcia $(1998 ; 2001)$ used computational turbulence modelling to determine the velocity and kinematic eddy viscosity distributions for one-dimensional flow through vegetation stems, and then one-dimensional diffusionconvection theory to determine the vertical suspended sediment concentration distributions. The transverse transfer of sediment from an unvegetated channel to adjacent vegetated zones is more complex, and requires description of the transverse distribution of velocity and the transverse eddy viscosity. Tsujimoto and Shimizu (1994) used a similar approach to that of Lopez and Garcia $(1998 ; 2001)$ to simulate the vertical and transverse distributions of suspended sediment concentration in simple and compound channels with vegetation zones in the cross-sections. Their model is able to predict deposition within a vegetation strip and its dependence on stem density.

An alternative to turbulence modelling for determining sediment diffusivity values is empirical evaluation for appropriate conditions. As part of a study of fine sediment settling within emergent vegetation, Elliott (2000) determined vertical diffusivities in an array of $6.4 \mathrm{~mm}$ diameter cylindrical rods set at a density of $3667 \mathrm{rods} / \mathrm{m}^{2}$ in a $6 \mathrm{~m}$ long, $0.29 \mathrm{~m}$ wide flume, by measuring concentration variations of injected dye. The diffusivity varied with velocity, slope and flow depth (Table 1), but the concentration distributions were consistent with constant values over the depth. Nepf (1999) used measurements of dye dispersion to determine transverse diffusivities in flows through arrays of cylindrical rods. She found transverse diffusivities to vary with stem characteristics and flow velocity according to:

$$
\frac{\varepsilon_{z}}{U d}=\alpha\left(C_{d} a d\right)^{1 / 3}+\left(\frac{\beta^{2}}{2}\right) a d
$$

where:

$U$ is the mean flow velocity

$d$ is the cylinder diameter

$\beta$ and $\alpha$ are scale factors

$C_{d}$ is the stem drag coefficient

$a\left(\mathrm{~m}^{-1}\right)$ is the projected plant area per unit volume.

\begin{tabular}{|c|c|c|c|}
\hline \multicolumn{4}{|c|}{$\begin{array}{c}\text { TABLE 1 } \\
\text { Vertical diffusivity values within stems } \\
\text { (Elliott, 2000) }\end{array}$} \\
\hline $\begin{array}{c}\text { Velocity } \\
(\mathbf{m m} / \mathbf{s})\end{array}$ & Slope & $\begin{array}{c}\text { Flow depth } \\
(\mathbf{m m})\end{array}$ & $\begin{array}{c}\text { Vertical diffusivity } \\
\left(\mathbf{m}^{2} / \mathbf{s}\right)\end{array}$ \\
\hline 8.1 & 0.00026 & 113 & $3.5 \times 10^{-6}$ \\
\hline 14.8 & 0.00055 & 122 & $14 \times 10^{-6}$ \\
\hline 24.7 & 0.00124 & 76 & $20 \times 10^{-6}$ \\
\hline
\end{tabular}

In this study, experiments were conducted to determine the influence of flow characteristics, grain size and stem density on the distribution of sediment deposited within emergent vegeta-

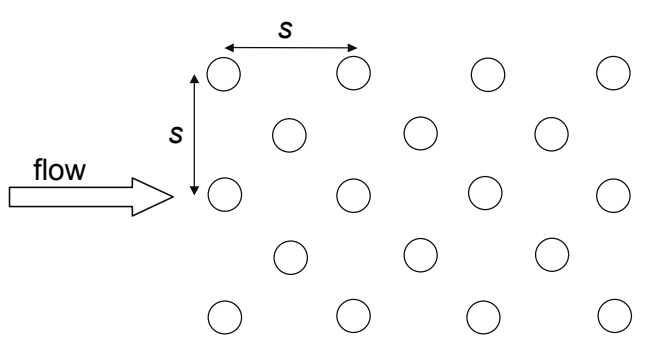

Figure 1

Arrangement of stems

tion stems from suspended transport. Although the deposition probability $(p)$ has a strong influence on deposition distribution (James, 1985; 1988), only a bed with $p=1.0$ was used. Both longitudinal and transverse transport situations were investigated. The two situations were simulated using appropriate formulations of the diffusion-convection model (Eq. (1)) to confirm their applicability and to enable inference of realistic values of sediment diffusivity.

\section{Experimental procedure}

Two series of flume experiments were carried out. In Series A, the longitudinal deposition patterns resulting from sediment transported in the direction of flow through emergent stems were investigated. In Series B, the deposition patterns resulting from the transverse diffusion of suspended sediment across the interface between longitudinal unvegetated and vegetated zones were investigated. Full details of the experimental programme are presented by Sharpe (2003).

\section{Series A: Longitudinal transport case}

The Series A experiments were conducted in a $0.38 \mathrm{~m}$ wide glass-sided flume with a $10 \mathrm{~m}$ tilting section set at a slope of 0.0019. Water was supplied from an overhead tank, with the discharge controlled by a valve upstream of the flume and measured by a V-notch weir at the downstream end. Vegetation stems were represented by $10 \mathrm{~mm}$ diameter vertical wooden rods set in timber boards above the water level in a staggered arrangement (Fig. 1), and extending over the full width and length of the flume. Three different stem spacings $(s)$ were tested $(127 \mathrm{~mm}$, $95 \mathrm{~mm}$ and $76 \mathrm{~mm}$ ), resulting in stem densities of $104 \mathrm{stems} / \mathrm{m}^{2}$, $194 \mathrm{stems} / \mathrm{m}^{2}$ and $312 \mathrm{stems} / \mathrm{m}^{2}$. The stem diameters and densities were selected to represent conditions typically occurring in South African rivers (James et al., 2001), with the intermediate density being the most representative. Sediment was introduced to the flume by a hopper-conveyor belt feeder located at the beginning of the tilting section. Sediment was dropped by the feeder onto the water surface, thus presenting a point source to the longitudinal-vertical plane. The sediment used was fine sand with a median grain size of $0.18 \mathrm{~mm}$ and $92.1 \%$ by weight between $0.075 \mathrm{~mm}$ and $0.300 \mathrm{~mm}$. The bed of the flume was covered with a spun filament ("Nomad") matting to create a fully absorbing boundary that trapped all settling sediment, i.e. with a deposition probability $(p)$ of 1.0 . The matting was cut into longitudinal strips to enable samples to be recovered from the central $0.144 \mathrm{~m}$ wide strip of the flume in sixteen $0.613 \mathrm{~m}$ longitudinal increments.

For each test, uniform flow was established by adjustment of a weir at the downstream end of the flume, and the sediment then introduced at a constant rate of $1.73 \mathrm{~g} / \mathrm{s}$ until a measurable quantity had deposited in the matting. The sediment trapped in 


\begin{tabular}{|c|c|c|c|c|}
\hline \multicolumn{5}{|c|}{ TABLE 2 } \\
\hline $\begin{array}{c}\text { Test } \\
\text { number }\end{array}$ & $\begin{array}{c}\text { Stem- } \\
\text { density } \\
\text { (stems/ } \\
\mathbf{m}^{2} \text { ) }\end{array}$ & $\begin{array}{c}\text { Dis- } \\
\text { charge } \\
\text { ( } / \text { /s) }\end{array}$ & $\begin{array}{c}\text { Flow- } \\
\text { depth } \\
\text { (mm) }\end{array}$ & $\begin{array}{c}\text { Duration } \\
\text { (min) }\end{array}$ \\
\hline A1 & 0 & 8.59 & 62 & 35 \\
A2 & 104 & 8.56 & 130 & 35 \\
A3 & 194 & 8.51 & 180 & 35 \\
A4 & 312 & 8.59 & 234 & 35 \\
A5 & 0 & 44.31 & 180 & 37 \\
A6 & 104 & 12.95 & 180 & 40 \\
A7 & 194 & 10.92 & 224 & 37 \\
A8 & 312 & 7.20 & 192 & 30 \\
*A9 & 0 & 25.67 & 124 & - \\
A10 & 104 & 16.11 & 222 & 35 \\
A11 & 194 & 6.42 & 132 & 24 \\
A12 & 312 & 4.60 & 125 & 17 \\
& & & & \\
B1 & 312 & 34.8 & 143 & 120 \\
B2 & 194 & 40.9 & 150 & 120 \\
B3 & 104 & 42.2 & 142 & 120 \\
B4 & 194 & 47.5 & 174 & 120 \\
B5 & 194 & 28.2 & 106 & 120 \\
\hline
\end{tabular}

*The deposition profile was not measured for this test

the centre matting strips was then washed out into separate pans, while that in the side strips was discarded. The sediment samples were dried in an oven and sieved through a stack of $0.300 \mathrm{~mm}$, $0.212 \mathrm{~mm}, 0.150 \mathrm{~mm}, 0.106 \mathrm{~mm}$ and $0.075 \mathrm{~mm}$ sieves, resulting in six size fractions. (Each size fraction is designated the size of its retaining sieve and therefore includes grains up to the next sieve size.) The mass of each size fraction obtained from a strip was measured and related to the location of the strip along the flume, producing a longitudinal mass deposition profile for each fraction. To facilitate comparison of distributions with different mass values, the profiles were expressed in relative terms by dividing each mass value by the maximum value.

In total 11 tests were performed, with experimental conditions as set out in Table 2. (Test A9 was carried out without sediment feed, to obtain hydraulic information only).

\section{Series B: Transverse transport case}

The Series B experiments were done in a $12 \mathrm{~m}$ long, $0.76 \mathrm{~m}$ wide channel lined with cement plaster and set on a slope of 0.0021 . The water supply, control and measurement arrangements were similar to those for Series A. The same artificial stems were used as in Series A, but in this case extended over only half the channel width. The matting was placed on the bed within the vegetated zone, where deposition was to be measured. At two measuring locations (about $5.1 \mathrm{~m}$ and $6.3 \mathrm{~m}$ downstream from the sediment feed location) the matting was cut into $39 \mathrm{~mm}$ wide longitudinal strips over a length of $0.306 \mathrm{~m}$. The sediment feeder was aligned to supply sediment over the unvegetated half of the channel width. The same sediment was used as in the Series A experiments.

For each test, uniform flow was established by adjusting a weir at the downstream end of the channel. Once uniform flow had been established, the transverse velocity profile at 0.4 times the flow depth from the bed within the unvegetated channel section was measured using a miniature propeller current meter at a location approximately $6 \mathrm{~m}$ downstream from the sediment feeder. Sediment was then introduced into the unvegetated channel section at $1.73 \mathrm{~g} / \mathrm{s}$ for approximately $2 \mathrm{~h}$. The transverse deposition profiles were determined following the same procedure as for the Series A experiments. Five experiments were performed, for the conditions listed in Table 2.

\section{Modelling}

The distribution of sediment deposited from suspension results from the spatial variation of concentration in transport and the interaction between material in transport and the bed. The distribution patterns of deposits for the experimental conditions described above can therefore be simulated by the diffusion-convection model for suspended transport (Eq. (1)) and the bed boundary condition (Eq. (2)). The experimental conditions represent special cases, and Eq. (1) can be simplified substantially to represent each of them. Two separate two-dimensional models have been formulated to represent the situations corresponding to the Series A and Series B situations, and solved in finite difference form in a spreadsheet (solution details are presented by Sharpe (2003)). The models were applied for the experimental conditions to establish their verisimilitude and to infer approximate values of vertical and transverse diffusivities (both assumed to be constant with depth).

\section{Longitudinal transport case}

For the longitudinal two-dimensional case the transverse $(z)$ components in Eq. (1) are ignored. The sediment is assumed to move downwards in the vertical $(y)$ direction at its settling velocity $\left(u_{y}=-w\right)$, and longitudinal diffusion is assumed to be negligible compared with convection $\left(\varepsilon_{x}=0\right)$. For steady state conditions the change in concentration with time is zero. Introducing these simplifications into Eq. (1) gives the two-dimensional diffusion-convection equation for suspended sediment transport under steady state conditions, i.e.:

$$
0=-u_{x} \frac{\partial C}{\partial x}+w \frac{\partial C}{\partial y}+\frac{\partial}{\partial y}\left(\varepsilon_{y} \frac{\partial C}{\partial y}\right)
$$

The boundary condition at the surface states that there is no net transport across the surface, and is written (e.g. James, 1985):

$$
\varepsilon_{y} \frac{\partial C}{\partial y}+w C=0
$$

When using a parabolic diffusivity profile (typical for flow in the absence of stems), the diffusivity at the surface is zero. In this case Eq. (5) shows that the concentration at the surface will also be zero. The concentration at the water surface was therefore always taken as zero.

The boundary condition near the bed, where suspended sediment transport begins, is represented by Eq. (2). The value of $p$ for the experimental conditions is 1.0

\section{Transverse transport case}

As for the longitudinal case, flow is assumed to be steady and the vertical velocity is represented by the sediment settling velocity. Because concentrations are required in a relative sense for individual cross sections, the longitudinal components in Eq. (1) are ignored. The longitudinally linear geometry implies zero transverse velocity $\left(u_{z}\right)$. Equation (1) then becomes:

$$
0=w \frac{\partial C}{\partial y}+\frac{\partial}{\partial z}\left(\varepsilon_{z} \frac{\partial C}{\partial z}\right)+\frac{\partial}{\partial y}\left(\varepsilon_{y} \frac{\partial C}{\partial y}\right)
$$


The boundary conditions at the surface and the bed are the same as for the longitudinal transport case. The boundary condition at a solid vertical surface is (e.g. James, 1985):

$$
\varepsilon_{z} \frac{\partial C}{\partial z}=0
$$

At the vegetation interface, the vertical suspended sediment concentration profile is determined by the unvegetated channel sediment transport characteristics, and is assumed to be defined by the equilibrium vertical concentration according to Rouse's (1937) equation:

$$
\frac{C}{C_{a}}=\left(\frac{D-y}{y} \frac{a}{D-a}\right)^{w / \alpha u s}
$$

where:

$C_{a}$ is a reference concentration at height $a$ above the bed $u_{*}^{a}$ is the shear velocity

$\kappa$ is the Von Karman constant.

Because the model is used to predict relative concentrations, $C_{a}$ is arbitrary and assumed to be 1.0. The vertical concentration profile assumed has an appreciable effect on the predicted deposition profiles; the measured deposition profiles were similar at the two sampling locations, however, suggesting that near equilibrium vertical concentration profiles had been attained.

\section{Results and discussion}

The distribution of sediment deposited from suspension is determined by the sediment, stem, bed and flow characteristics. The experimental results enable the influences of sediment grain size, stem density and flow depth to be demonstrated. The sediment diffusivity could not be measured in these experiments, but approximate values were determined by using it as a calibrating parameter to fit simulated and experimental results.

\section{Longitudinal transport case}

The measured longitudinal deposition profiles show a characteristic form, with mass deposited increasing to a peak some distance downstream from the feed location and then decreasing gradually further downstream. The initial increase is caused by the feed being a point source at the water surface, so that settling through the full flow depth (under the influence of the longitudinal flow velocity) is required before any deposition occurs on the bed. The subsequent decrease is a result of the deposition up to any location reducing sediment availability further downstream.

The effect of grain size on the relative deposition distribution is illustrated in Fig. 2 by the results for the different size fractions (represented by the retaining sieve sizes) for Test A7. The particles are all settling under the same flow velocity, flow depth, stem density and turbulence conditions. Because settling velocity is proportional to grain size, the distribution peak occurs closer to the feed location for the larger grains. The smaller the grain size the more gradual the rise to the peak, and the more extensive is the deposit, because the slower settling leads to greater travel distance before reaching the bed.

Figure 3 shows the effect of discharge and flow depth on the distribution of deposition. The nature of vegetative resistance is such that average flow velocity does not increase with depth as for an unvegetated channel (James et al., 2001). An increase in discharge is therefore manifest as an increase in depth rather

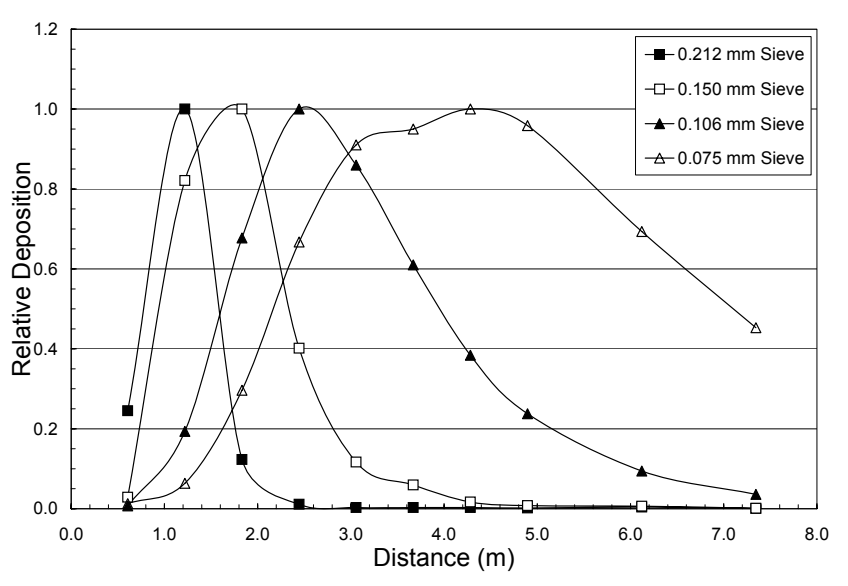

Figure 2

Influence of sediment size on longitudinal deposition profile (medium density stems, flow rate $=10.92 \mathrm{l} / \mathrm{s}$ )

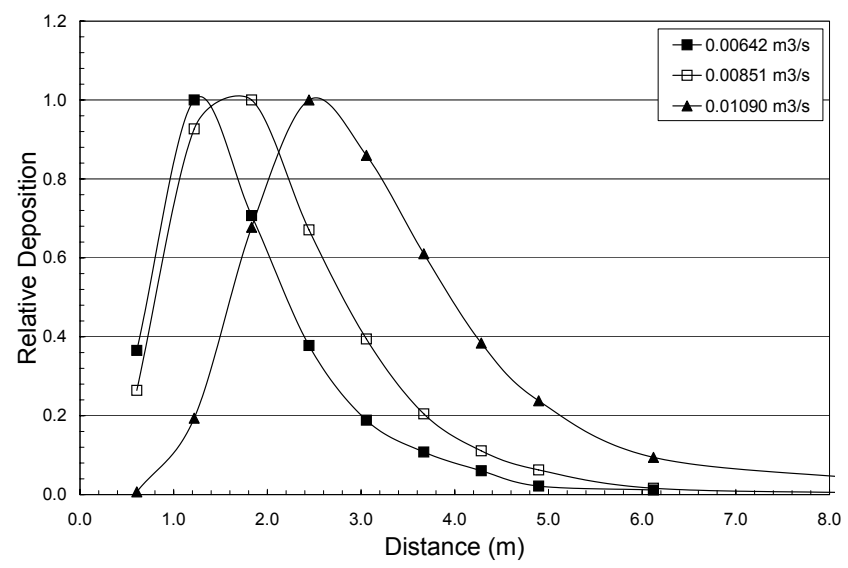

Figure 3

Influence of discharge and flow depth on longitudinal deposition profile (0.106 $\mathrm{mm}$ sieve sediment size, medium density stems)

than velocity. The relative deposition distributions for one grain size fraction ( $0.106 \mathrm{~mm}$ retaining sieve) are shown in Fig. 3 for Tests A11, A3 and A7, all with the same stem density, but different discharges and hence different flow depths, but similar velocities. The upstream shift of the peak location and the slight steepening of the rising limb with reducing discharge are caused by the shorter vertical settling distance with similar flow velocities. The corresponding reduction in spread of the distribution can be attributed to the reduced opportunity for vertical diffusion in the shorter water column.

The average flow velocity through emergent vegetation decreases in inverse proportion to the square root of the stem density (James et al., 2004). For a constant flow depth, the effect of a change in stem density on the relative sediment deposition distribution would therefore be expected to lessen with increasing stem densities. This is supported by the data, for example for the $0.106 \mathrm{~mm}$ retaining sieve grain size fraction in Tests A5, A6, A3 and A8, with different stem densities but similar flow depths of around $180 \mathrm{~mm}$ (Fig. 4). As flow velocity is reduced by increased stem density, the sediment settles to the bed over a shorter distance. It is clear that the introduction of stems has a strong influence on deposition compared with the unvegetated channel case, but the incremental effect diminishes so there is little difference for a $60 \%$ increase in density from Test A3 to Test A8. 
Turbulent velocity fluctuations were not measured in this study, and so no direct estimates of eddy viscosity within stems could be obtained. Approximate values of vertical sediment diffusivity were, however, inferred by fitting simulated relative deposition distributions to those measured for all the Series A experimental conditions and grain size fractions. Settling velocities for the different grain sizes were determined from the graphical relationship presented by Graf (1971) for natural sediments. The veracity of the model was first established by reproducing the distribution patterns for the two experiments with no stems, assuming the vertical sediment diffusivity to be related to the conventional parabolic vertical distribution of eddy viscosity by (Graf, 1971):

$$
\varepsilon_{y}=\beta v_{t}=\beta \kappa u_{*} \frac{y}{D}(D-y)
$$

where:

$\beta$ is a constant of proportionality

$v_{t}$ is eddy viscosity

$\kappa$ is the Von Karman constant

$u$ is the shear velocity

$y$ is the vertical co-ordinate

$D$ is the flow depth.

The value for $\beta$ was determined using an empirical equation (Eq. (10)) developed by Graf and Cellino (2002):

$$
\beta=\frac{3}{10}+\frac{3}{4} \frac{w}{u_{*}}
$$

In each case the model was used to simulate deposit distributions for sediment sizes corresponding to the sieve sizes, hence producing upper and lower bounds for the size fractions recovered in the experiments. The general forms of the deposition profiles, and the influence of settling velocity were well reproduced, although the longitudinal distance travelled by each size fraction was underestimated (Fig. 5 shows the simulations for Test A1). This may indicate that longitudinal diffusion is not insignificant in comparison with longitudinal convection, as assumed in Eq. (4).

For the cases with stems, the diffusivity was adjusted to obtain satisfactory agreement between measured and simulated deposit distributions. Some preliminary simulations with uniform and parabolic vertical distributions of diffusivity showed that the results were insensitive to diffusivity distribution, and a uniform distribution (as suggested by Elliott (2000)) was used for all subsequent simulations. As for the cases with no stems, the forms of the deposit distributions and their variation with settling velocity were well reproduced (Fig. 6 for Test A12, for example). For all cases, constant values of vertical diffusivity of $40 \times 10^{-6} \mathrm{~m}^{2} / \mathrm{s}$ or $50 \times 10^{-6} \mathrm{~m}^{2} / \mathrm{s}$ produced reasonable fits. These values are about an order of magnitude less than the depth-averaged values for the cases with no stems, according to the parabolic distribution. For the range of experimental conditions, diffusivity shows no apparent dependence on stem density, flow velocity or flow depth. The values measured by Elliott (2000) (Table 1) were lower than obtained here. His stem diameters were smaller, stem densities much higher and velocities much lower, however, suggesting that diffusivity is increased by higher flow velocities and lower stem densities.

\section{Transverse transport case}

The measured transverse distributions of deposited sediment within a longitudinal vegetation strip adjacent to an

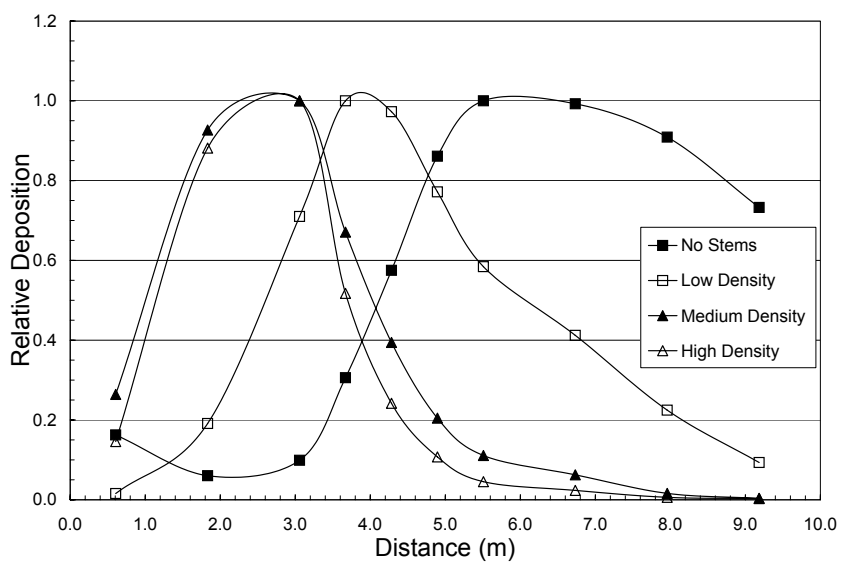

Figure 4

Influence of stem density on longitudinal deposition profile (0.106 $\mathrm{mm}$ sieve sediment size, flow depth $\approx 180 \mathrm{~mm}$ )

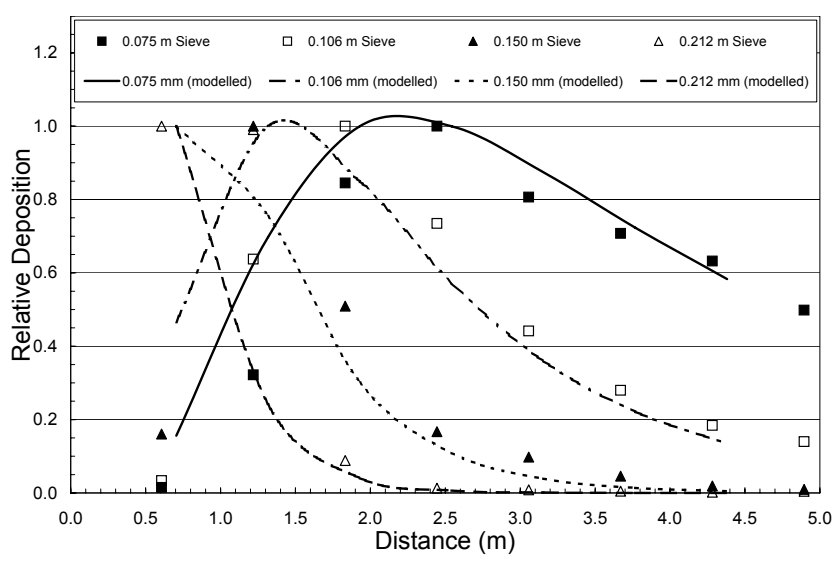

Figure 5

Measured and simulated longitudinal deposition profiles with no stems and flow depth $=62 \mathrm{~mm}$

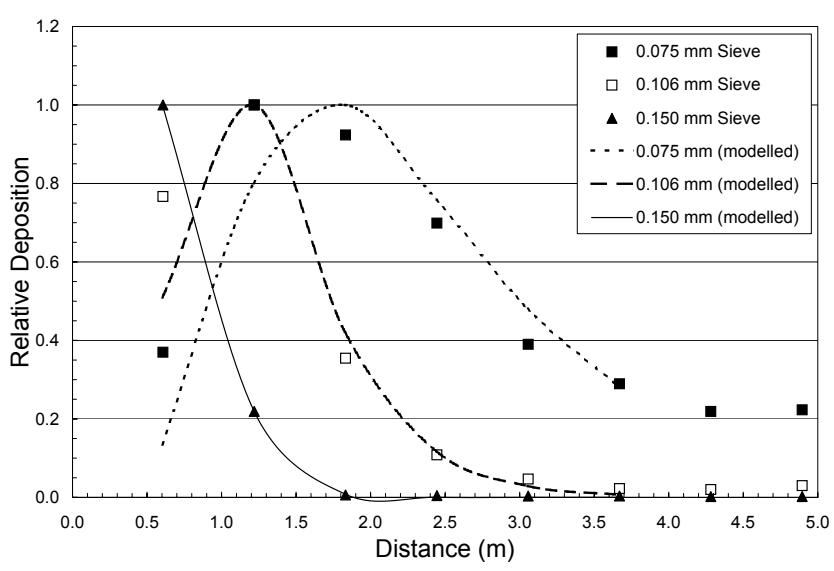

Figure 6

Measured and simulated longitudinal deposition profiles with high stem density and flow depth $=125 \mathrm{~mm}$

unvegetated channel all show peak deposition rates close to the vegetation interface, with deposition decreasing consistently with distance from the interface. As there is no transverse convection, this pattern results from transverse diffusion, associated with the velocity gradient through the transition zone between the unaffected flow in the unvegetated and vegetated zones. 


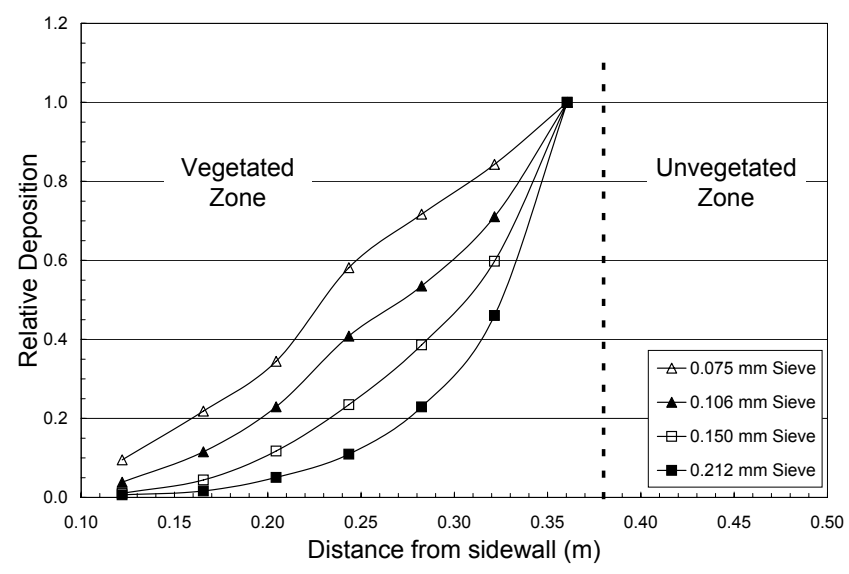

Figure 7

Influence of sediment size on transverse deposition profile (medium density stems, flow depth $=150 \mathrm{~mm}$ )

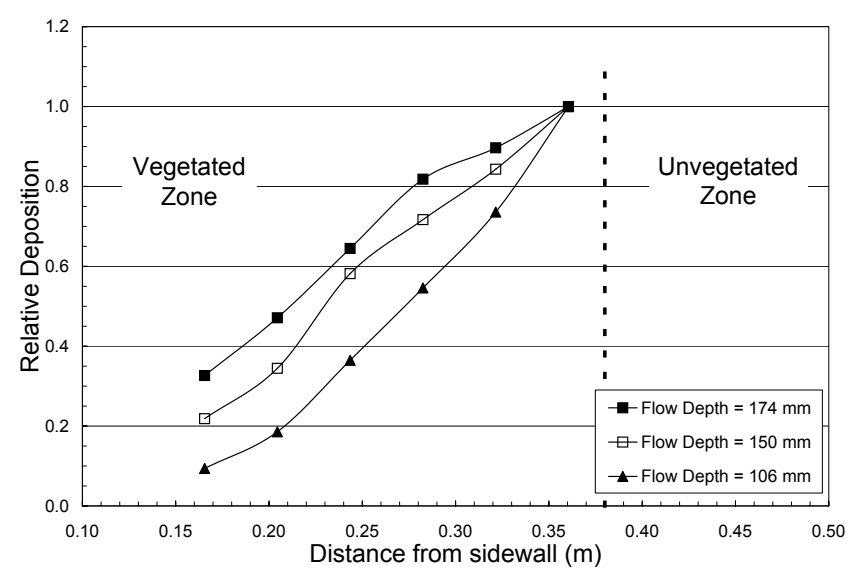

Figure 8

Influence of flow depth on transverse deposition profile (medium stem density, $0.075 \mathrm{~mm}$ sieve sediment size)

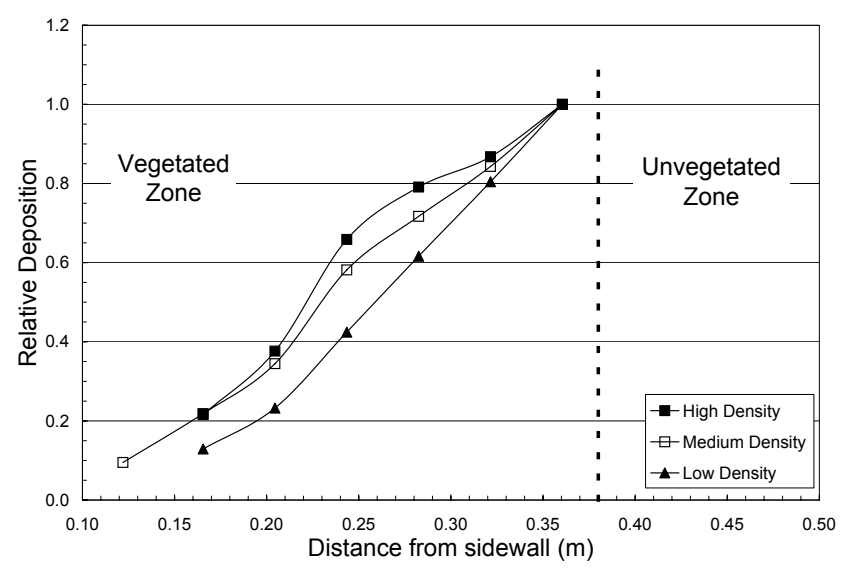

Figure 9

Influence of stem density on transverse deposition profile (flow depth $\approx 145 \mathrm{~mm}, 0.075 \mathrm{~mm}$ sieve sediment size)

The transverse deposit distribution is affected significantly by the sediment grain size (Fig. 7). The more extensive deposits for finer fractions result from their lower settling velocities and relatively larger concentrations higher in the flow at the interface and hence longer settling times and distances while being transferred transversely by diffusion. Transverse deposits in instream vegetation strips would therefore be expected to be

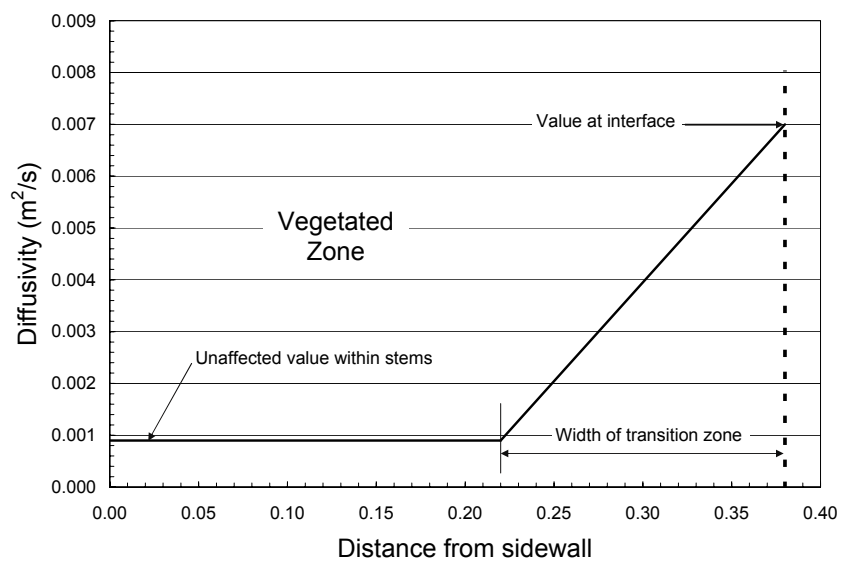

Figure 10

Assumed form of transverse diffusivity profile

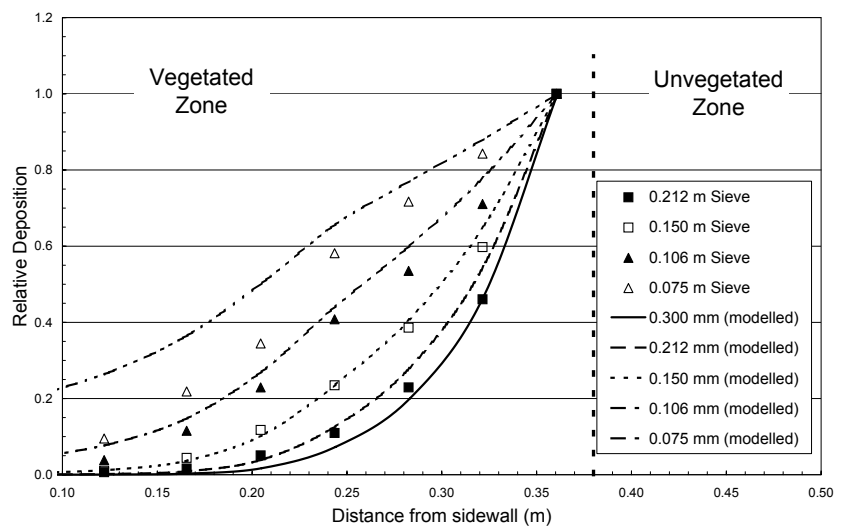

Figure 11

Measured and simulated transverse deposition profiles with high density stems and flow depth $=143 \mathrm{~mm}$

larger and coarser close to the unvegetated channel boundary and to become smaller and finer away from the boundary, similar to flood plain deposits (James, 1985).

The extent of transverse deposition is also influenced by flow depth, as shown in Fig. 8 for one size fraction and a common stem density. Deposits are more extensive with deeper flows because of greater settling distances during transverse diffusion. Greater flow depths are also associated with greater discharges and hence greater velocities in the unvegetated channel. As velocity within the stems does not increase with flow depth, larger velocity gradients and hence greater diffusivities occur with deeper flows, leading to more extensive deposits. The increase of transverse diffusivity with flow depth was confirmed by application of the transverse diffusion-convection model.

Stem density increases the extent of the transverse deposit, although this effect appears to lessen with increasing density (Fig. 9, for one size fraction and similar flow depths). This is attributed to the greater rate of transfer across the vegetation interface, as both vertical and transverse diffusivities within the stems were found to be similar for all densities in the model applications. The transverse diffusivity through the transition zone is, however, increased substantially by stem density through the increased velocity difference between the two zones.

The transverse diffusion-convection model was used to reproduce the experimental results and hence infer transverse diffusivity characteristics in the transition zone within the stems. The transverse diffusivity was assumed to vary linearly from the vegetation interface to the end of the transition 


\begin{tabular}{|c|c|c|c|c|c|c|}
\hline \multicolumn{7}{|c|}{ TABLE 3 } \\
\hline $\begin{array}{c}\text { Test } \\
\text { number }\end{array}$ & $\begin{array}{c}\text { Stem density } \\
\left(\mathbf{s t e m s} / \mathbf{m}^{2}\right)\end{array}$ & $\begin{array}{c}\text { Flow } \\
\text { depth } \\
(\mathbf{m m})\end{array}$ & $\begin{array}{c}\text { Transition } \\
\text { zone width } \\
(\mathbf{m})\end{array}$ & $\begin{array}{c}\text { Diffusivity } \\
\text { at stem } \\
\text { interface } \\
\left(\mathbf{m}^{2} / \mathbf{s}\right)\end{array}$ & $\begin{array}{c}\text { Diffusivity } \\
\text { in stems } \\
(\mathbf{s i m u l a t e d}) \\
\left(\mathbf{m}^{2} / \mathbf{s}\right)\end{array}$ & $\begin{array}{c}\text { Diffusivity } \\
\text { in stems } \\
\left(\begin{array}{c}\text { Eq. } \\
(\mathbf{3}))\end{array}\right. \\
\left(\mathbf{m}^{2} / \mathbf{s}\right)\end{array}$ \\
\hline B1 & 312 & 143 & 0.16 & $11 \times 10^{-3}$ & $0.9 \times 10^{-3}$ & $0.30 \times 10^{-3}$ \\
\hline B2 & 194 & 150 & 0.16 & $7 \times 10^{-3}$ & $0.9 \times 10^{-3}$ & $0.32 \times 10^{-3}$ \\
\hline B3 & 104 & 142 & 0.16 & $4 \times 10^{-3}$ & $0.9 \times 10^{-3}$ & $0.36 \times 10^{-3}$ \\
\hline B4 & 194 & 174 & 0.16 & $8 \times 10^{-3}$ & $0.9 \times 10^{-3}$ & $0.32 \times 10^{-3}$ \\
\hline B5 & 194 & 106 & 0.12 & $5 \times 10^{-3}$ & $0.9 \times 10^{-3}$ & $0.32 \times 10^{-3}$ \\
\hline
\end{tabular}

zone within the stems, and then remain constant throughout the remaining width of the vegetation (Fig. 10). The variation of transverse diffusivity is therefore characterised by the values at the interface and beyond the transition zone and the width of the transition zone. These three parameters were adjusted to obtain agreement between measured and simulated deposit distributions. The resulting values are presented in Table 3 , and an example of the simulated results in Fig. 11. (This figure includes a modelled curve for a sediment size of $0.300 \mathrm{~mm}$ to indicate the upper bound for the experimental fraction designated by the sieve size of $0.212 \mathrm{~mm}$ ).

The results suggest that diffusivities within the stems but beyond the transition zone were independent of stem density and flow depth for the range of conditions tested. The values of transverse diffusivity determined by the simulations were of the same order of magnitude but somewhat greater than predicted by Nepf's (1999) equation (Eq. (3)), with the flow velocity predicted using the equation proposed by James et al. (2001) (Table 3). The discrepancy could be due to the fact that Nepf determined her values from dye diffusion measurements and the diffusion of sediment grains could be greater because of the circulatory character of the turbulence induced by the stems (Singamsetti, 1966). Within the transition zone, the transverse diffusivity was found to increase with stem density and flow depth. The values at the interface varied linearly with stem density and flow depth over the range of experimental conditions.

The satisfactory performance of the diffusion-convection model formulations for both the longitudinal and transverse transport cases for the experimental conditions suggests their suitability for describing deposition patterns in more realistic cases. The most significant modification required would be specification of a deposition probability $(p)$ for the bed boundary condition expressed by Eq. (2). James (1988) used Einstein's (1950) entrainment probability to determine this parameter for unvegetated channel flow, but this would require more detailed study of near-bed flow conditions for application in the presence of stems.

\section{Conclusions}

Emergent vegetation stems promote deposition of sediment from suspension by reducing flow velocity and vertical sediment diffusivity. Vertical sediment diffusivity is reduced substantially by the presence of stems (by approximately an order of magnitude for the conditions investigated here). The longitudinal distribution of deposits becomes more localised and less extensive with increasing grain size and stem density and decreasing flow depth.

The shear zone along the interface between longitudinal vegetation strips and unvegetated flow promotes ingress of sediment into the vegetation by locally enhancing transverse sedi- ment diffusivity. Transverse diffusivity appears to be increased slightly by stems beyond the shear zone, but by about an order of magnitude within the shear zone. The transverse extent of deposits within the stems increases with flow depth and stem density and decreases with sediment grain size.

The diffusion-convection model realistically predicts the distribution of deposits for both the longitudinal and transverse cases (provided diffusivity values and deposition probability are known), and reflects the relative sensitivities of sediment movement to the effects of stems on flow velocity and sediment diffusivity. The slight underestimation of longitudinal deposit extent for the experimental conditions may indicate that longitudinal diffusion is not negligible in comparison with longitudinal convection for the cases investigated.

\section{Acknowledgements}

This material is based upon work supported by the National Research Foundation (NRF). Any opinion, findings and conclusions or recommendations expressed in this material are those of the authors and therefore the NRF does not accept any liability in regard thereto.

\section{References}

EINSTEIN HA (1950) The bed-load function for sediment transportation in open channel flows. Soil Conservation Service Tech. Bull. US Dept. of Agric., Washington, DC.

ELLIOTT AH (2000) Settling of fine sediment in a channel with emergent vegetation. J. Hydraul. Eng. 126 (8) 570-577.

GRAF WH (1971) Hydraulics of Sediment Transport. McGraw-Hill, New York.

GRAF WH and CELLINO M (2002) Suspension flows in open channels; experimental study. J. Hydraul. Res. 40 (4) 435-447.

JAMES CS (1985) Sediment transfer to overbank sections. J. Hydraul. Res. 23 (5) 435-452.

JAMES CS (1988) Use of the Einstein entrainment function for predicting selective entrainment and deposition of heavy minerals. Water SA 14 (4) 219-228.

JAMES CS, BIRKHEAD AL, JORDANOVA AA and O'SULLIVAN JJ (2004) Flow resistance of emergent vegetation. J. Hydraul. Res. 42 (4) 390-398.

JAMES CS, BIRKHEAD AL, JORDANOVA AA, KOTSCHY, KA, NICOLSON CR and MAKOA, MJ (2001) Interaction of Reeds, Hydraulics and River Morphology. WRC Report No. 856/1/01. Water Research Commission, Pretoria, South Africa.

LOPEZ F and GARCIA M (1998) Open-channel flow through simulated vegetation: Suspended sediment transport modelling. Water Resour. Res. 34 (9) 2341-2352.

LOPEZ F and GARCIA M (2001) Mean flow and turbulence structure of open-channel flow through non-emergent vegetation. J. Hydraul. Eng. 127 (5) 392-402.

NEPF HM (1999) Drag, turbulence, and diffusion in flow through emergent vegetation. Water Resour. Res. 35 (2) 479-489. 
ROUSE H (1937) Modern conceptions of the mechanics of turbulence, Trans. Am. Soc. Civil Eng. 102 463-543.

SHARPE RG (2003) Suspended Sediment Transport through NonSubmerged Reeds. M.Sc. (Eng.) dissertation, University of the Witwatersrand, Johannesburg, South Africa.

SINGAMSETTI SR (1966) Diffusion of sediment in a submerged jet. J. Hydraul. Div. ASCE 92 (HY2) 153-169.
TSUJIMOTO T and SHIMIZU Y (1994) Flow and suspended sediment in a compound channel with vegetation. Proc. $1^{\text {st }}$ Int. Symp. on Habitat Hydraulics. Held in Trondheim, Norway, August. 357-370.

VAN RIJN LC (1982) Sediment transport, Part II: Suspended load transport. J. Hydraul. Eng. 110 (11) 1613-1641. 